\title{
Entanglement renormalization for interacting field theories
}

\author{
J. J. Fernández-Melgarejo $\odot,{ }^{1, *}$ J. Molina-Vilaplana, ${ }^{2, \dagger}$ and E. Torrente-Lujan ${ }^{1, \$}$ \\ ${ }^{1}$ Universidad de Murcia, Campus de Espinardo, E-30100 Murcia, Spain \\ ${ }^{2}$ Universidad Politécnica de Cartagena, UPCT, 30202 Cartagena, Spain
}

(Received 27 April 2019; published 26 September 2019)

\begin{abstract}
A general method to build the entanglement renormalization (cMERA) for interacting quantum field theories is presented. We improve upon the well-known Gaussian formalism used in free theories through a class of variational non-Gaussian wave functionals for which expectation values of local operators can be efficiently calculated analytically and in a closed form. The method consists of a series of scale-dependent nonlinear canonical transformations on the fields of the theory under consideration. Here, the $\lambda \phi^{4}$ and the sine-Gordon scalar theories are used to illustrate how nonperturbative effects far beyond the Gaussian approximation are obtained by considering the energy functional and the correlation functions of the theory.
\end{abstract}

DOI: 10.1103/PhysRevD.100.065025

\section{INTRODUCTION}

In recent years, tensor networks, a new and powerful class of variational states, have proved to be very useful in addressing both static and dynamical aspects of a wide number of interacting many-body systems. They represent a class of systematic variational ansätze which, through the Rayleigh-Ritz variational principle, provide an elegant approximation to the ground state of an interacting theory by systematically identifying those degrees of freedom that are actually relevant for observable physics. These variational ansätze are nonperturbative and can be applied both in the lattice and in the continuum. As an example, the multiscale entanglement renormalization Ansatz (MERA), a variational real-space renormalization scheme on the quantum state, represents the wave function of the quantum system at different length scales [1].

A continuous version of MERA, known as cMERA, was proposed in [2] for free field theories. It consists of building a scale-dependent representation of the ground state wave functional through a scale-dependent linear canonical transformation of the fields of the theory. Namely, the renormalization in scale is generated by a quadratic operator, and thus, the resulting state is given by a Gaussian wave functional. Despite this fact obviously

\footnotetext{
*jj.fernandezmelgarejo@um.es

javi.molina@upct.es

torrente@cern.ch
}

Published by the American Physical Society under the terms of the Creative Commons Attribution 4.0 International license. Further distribution of this work must maintain attribution to the author(s) and the published article's title, journal citation, and DOI. Funded by SCOAP. limits the interest of this trial state for interacting quantum field theories (QFT), the Gaussian ansatz has been used in cMERA and correctly reproduces correlation functions and entanglement entropy in free field theories $[3,4]$. Furthermore, as the Gaussian cMERA is currently studied as a possible realization of holography [5-10], it is timely to develop interacting versions of cMERA in order to advance in this program. In [11], the Gaussian cMERA was applied to interacting bosonic and fermionic field theories. In [12], authors developed some techniques to build systematic perturbative calculations of cMERA circuits but restricted to the weakly interacting regime.

Our aim here is to provide a nonperturbative method to build truly non-Gaussian cMERA wave functionals for interacting QFTs. A justifiable way of doing so would be to formulate a perturbative expansion for which the Gaussian wave function appears in its first order [13-16]. Unfortunately, with these methods, expectation values of operators cannot be calculated exactly and must be approximated by an additional series expansion. On the contrary, our approach clings to the variational method, but using a more elaborated class of trial wave functionals. Here, we use a set of nonlinear canonical transformations (NLCT) [17-21] to build a set of scale-dependent extensive functionals which are certainly non-Gaussian. Remarkably, with this prescription, observables can be analytically calculated in a closed form. We illustrate the method by considering the self-interacting $\lambda \phi^{4}$ scalar theory and the sine-Gordon model in $(d+1)$ dimensions. For $d=1$, these theories, do not exhibit any issue when renormalization is considered, and thus the non-Gaussian cMERA lies on a solid ground. In addition, our variational procedure adds up a much larger class of Feynman diagrams than the usual "cactus"-like ones which are captured by the Gaussian 
approach [22]. Therefore, we are certainly generalizing the variational approach in QFT to non-Gaussian trial states in the canonical formalism.

\section{GAUSSIAN cMERA}

cMERA [2,3] is a real-space renormalization group procedure on the quantum state that builds a scaledependent wavefunctional $\Psi[\phi, u]$,

$$
\Psi[\phi, u]=\left\langle\phi \mid \Psi_{u}\right\rangle=\left\langle\phi\left|\mathcal{P} e^{-i \int_{u_{\mathrm{IR}}}^{u}\left(K\left(u^{\prime}\right)+L\right) d u^{\prime}}\right| \Omega_{\mathrm{IR}}\right\rangle,
$$

where $u$ parametrizes the scale of the renormalization. (1) contains the path-ordered exponential of the dilatation operator $L$ and the generating operator $K\left(u^{\prime}\right)$. The renormalization scale parameter $u$ in cMERA is usually taken to be in the interval $\left[u_{\mathrm{IR}}, u_{\mathrm{UV}}\right]=(-\infty, 0] . u_{\mathrm{UV}}=u_{\epsilon}$ is the scale at the UV cutoff $\epsilon$, and the corresponding momentum space UV cutoff is $\Lambda=1 / \epsilon . u_{\mathrm{IR}}=u_{\xi}$ is the scale in the IR limit, where $\xi$ is a long-wavelength correlation length. The state $\left|\Psi_{\mathrm{UV}}\right\rangle$ is the ground state of a quantum field theory. The $L$-invariant state $\left|\Omega_{\mathrm{IR}}\right\rangle$ is a Gaussian state with no entanglement between spatial regions. The cMERA Hamiltonian evolution generates translations along the cMERA parameter $u$. The term $K(u)$ in the cMERA-Hamiltonian is called the entangler operator and the only variational parameters of the ansatz are those which parametrize it. For free scalar theories, $K(u)$ is the quadratic operator given by $[2,3]$

$$
K(u)=\frac{1}{2} \int_{\mathbf{p}} g_{0}(p, u)[\phi(\mathbf{p}) \pi(-\mathbf{p})+\pi(\mathbf{p}) \phi(-\mathbf{p})],
$$

where $p \equiv|\mathbf{p}|$ and $\int_{\mathbf{p}} \equiv \int(2 \pi)^{-d} d^{d} p$ with $d$, the spatial dimensions of the theory. The conjugate momentum of the field $\phi(\mathbf{p})$ is $\pi(\mathbf{p})=-i \bar{\delta} / \delta \phi(-\mathbf{p})$, such that $[\phi(\mathbf{p}), \pi(\mathbf{q})]=$ $i \bar{\delta}(\mathbf{p}+\mathbf{q})$, with $\bar{\delta}(\mathbf{p}) \equiv(2 \pi)^{d} \delta(\mathbf{p})$. The function $g_{0}(p, u)$ in (2) is the only variational parameter to optimize in the Gaussian cMERA. This function factorizes as $g_{0}(p ; u)=$ $g_{0}(u) \Gamma(p / \Lambda)$ where $\Gamma(x) \equiv \Theta(1-|x|)$ and $\Theta(x)$ is the Heaviside step function; $g_{0}(u)$ is a real-valued function and $\Gamma(p / \Lambda)$ implements a high-frequency cutoff such that $\int_{\mathbf{p}} \equiv \int_{\mathbf{p}}^{\Lambda}$. Choosing $\left|\Omega_{\mathrm{IR}}\right\rangle$ as [3]

$$
\left(\sqrt{\omega_{\Lambda}}\left(\phi(\mathbf{p})-\chi_{0}\right)+\frac{i}{\sqrt{\omega_{\Lambda}}} \pi(\mathbf{p})\right)\left|\Omega_{\mathrm{IR}}\right\rangle=0,
$$

for all $\mathbf{p}$, where $\omega_{\Lambda}=\sqrt{\Lambda^{2}+m^{2}}$ with $m$ the mass of the particles in the free theory, it is possible to show that the cMERA ansatz with a quadratic entangler is equivalent to the Gaussian wave functional given by

$$
\Psi[\phi ; u]_{S G}=N e^{-\frac{1}{2} \int_{\mathbf{p}}\left(\phi(\mathbf{p})-\chi_{0}\right) F^{-1}(p ; u)\left(\phi(-\mathbf{p})-\chi_{0}\right)},
$$

where $\chi_{0}=\left\langle\Psi_{S G}(u=0)|\phi(x)| \Psi_{S G}(u=0)\right\rangle$ and the relation between the scale-dependent Gaussian kernel $F(p ; u)$ and the variational cMERA parameter $g_{0}(p, u)$ is given by [11]

$$
F^{-1}(p ; u)=\omega_{\Lambda} e^{2 \int_{0}^{u} d u^{\prime} g_{0}\left(p e^{-u^{\prime}}, u^{\prime}\right)},
$$

with $F(p ; 0)=\left(p^{2}+m^{2}\right)^{-1 / 2}$.

We note that $\Psi[\phi ; u]_{S G}=U_{S} \Psi_{G}[\phi ; u]$, where the operator that shifts the argument of any functional (and specifically the Gaussian wave functional) by a constant $\chi_{0}$, is given by $U_{S}=e^{O_{S}}$ with $O_{S}=-\int_{\mathbf{p}} \chi_{0} \delta / \delta \phi(-\mathbf{p})$. Then, defining $U_{G}\left(u_{1}, u_{2}\right) \equiv \mathcal{P} e^{-i \int_{u_{2}}^{u_{1}} d u(K(u)+L)}$, the Gaussian state is given by $\Psi[\phi ; u]_{G}=\left\langle\phi\left|U_{G}\left(u, u_{\mathrm{IR}}\right)\right| \Omega_{\mathrm{IR}}\right\rangle$.

Finally, we remark that the Gaussian cMERA ansatz may be also understood as the set of scale-dependent linear transformation of the fields given by

$$
\begin{gathered}
U_{G}(0, u)^{-1} \phi(\mathbf{p}) U_{G}(0, u)=e^{-f(p, u)} e^{-\frac{u}{2} d} \phi\left(\mathbf{p} e^{-u}\right), \\
U_{G}(0, u)^{-1} \pi(\mathbf{p}) U_{G}(0, u)=e^{f(p, u)} e^{-\frac{u}{2} d} \pi\left(\mathbf{p} e^{-u}\right),
\end{gathered}
$$

with $f(p, u)=\int_{0}^{u} d u^{\prime} g_{0}\left(p e^{-u^{\prime}}, u^{\prime}\right)$.

\section{NON-GAUSSIAN cMERA}

In QFT, trial states created by introducing polynomial corrections to a Gaussian state correspond to a finite number of particles and those are suppressed in the thermodynamic limit. Thus, in going beyond the Gaussian ansatz, it is necessary to use a class of variational extensive states for which the energy density does not depend on the volume. Following [17,18,21], we build extensive non-Gaussian trial states considering wave functionals of the form

$$
\Psi_{N G}[\phi]=U_{N G} \Psi_{G}[\phi]=\exp (\mathcal{B}) \Psi_{G}[\phi],
$$

where the $N G$ subscript refers to non-Gaussian, $\Psi_{G}[\phi]$ is a normalized Gaussian wave functional and $U_{N G}=\exp (\mathcal{B})$, with $\mathcal{B}^{\dagger}=-\mathcal{B}$, an anti-Hermitian operator that, for the moment, it may add new variational parameters, in addition to those in the Gaussian wave functional. The expectation value of any operator $\mathcal{O}(\phi, \pi)$ in these states amounts to the calculation of a Gaussian expectation value for the transformed operator $\tilde{\mathcal{O}}=U_{N G}^{\dagger} \mathcal{O} U_{N G}$, i.e., $\left\langle\Psi_{N G}|\mathcal{O}(\phi, \pi)| \Psi_{N G}\right\rangle=$ $\left\langle\Psi_{G}\left|U_{N G}^{\dagger} \mathcal{O}(\phi, \pi) U_{N G}\right| \Psi_{G}\right\rangle$. The transformed operator $\tilde{\mathcal{O}}$ is straightforwardly built once the transformations

$$
\tilde{\phi}(\mathbf{p})=U_{N G}^{\dagger} \phi(\mathbf{p}) U_{N G}, \quad \tilde{\pi}(\mathbf{p})=U_{N G}^{\dagger} \pi(\mathbf{p}) U_{N G},
$$

are known. The transformation on the operator $\mathcal{O}$ generated by $\mathcal{B}$ is given by the Hadamard's lemma in terms of a series of nested commutators [27]

$$
\tilde{\mathcal{O}}=\operatorname{Ad}_{\mathcal{B}}(\mathcal{O})=e^{\operatorname{ad}_{\mathcal{B}}} \mathcal{O} .
$$


It can be seen that a suitable choice of $\mathcal{B}$, while leading to a non-Gaussian trial state, can indeed truncate the commutator expansion, thus reducing the calculation of expectation values of functionals to a finite number of Gaussian expectation values [28]. The exponential form of the transformation ensures the correct extensive volume dependence of observables such as the energy of the system. In addition, as $U_{N G}$ is unitary, the normalization of the state is preserved. The operator $\mathcal{B}$ consists of a product of $\pi$ 's and $\phi$ 's, which is given by

$$
\mathcal{B}=-s \int_{\mathbf{p q}_{1} \cdots \mathbf{q}_{m}} h\left(\mathbf{p}, \mathbf{q}_{1}, \ldots, \mathbf{q}_{m}\right) \frac{\delta}{\delta \phi(-\mathbf{p})} \phi\left(\mathbf{q}_{1}\right) \ldots \phi\left(\mathbf{q}_{m}\right),
$$

where $h\left(\mathbf{p}, \mathbf{q}_{1}, \ldots, \mathbf{q}_{m}\right)=g\left(p, q_{1}, \ldots, q_{m}\right) \bar{\delta}\left(\mathbf{p}+\mathbf{q}_{1}+\cdots+\mathbf{q}_{m}\right)$, $s$ is a variational parameter, $g\left(p, q_{1}, \ldots, q_{m}\right)$ is a variational function that must be optimized upon energy minimization and $m \in \mathbb{N}$. The other variational parameter is the kernel $F(p)$ entering the Gaussian wave functional. The explicit dependence of these parameters on the interaction couplings of a theory is established through energy minimization. This will be discussed later for some concrete examples. The function $g\left(p, q_{1}, \ldots, q_{m}\right)$ is symmetric under the exchange of $q_{i}$ 's, it must ensure the anti-Hermiticity of $\mathcal{B}$ and is constrained to satisfy $g\left(p, p, q_{2}, \ldots, q_{m}\right)=0$ and $g\left(p, q_{1}, \ldots, q_{m}\right) g\left(q_{i}, k_{1}, \ldots, k_{m}\right)=0$, for $i=1, \ldots, m$. This constraint ensures that the multiple commutator series in (10) terminates after the first nontrivial term. Such procedure yields a variational approximation to the calculation of observables in an interacting theory which improves upon the Gaussian ansatz. The parameter $s$ is a truly nonGaussian tracking parameter which shows the deviation of any observable from the Gaussian case.

The action of $U_{N G}$ on the canonical field operators is given by

$\tilde{\phi}(\mathbf{p})=\phi(\mathbf{p})+s \Phi(\mathbf{p}), \quad \tilde{\pi}(\mathbf{p})=\pi(\mathbf{p})+s \Pi(\mathbf{p})$,

with

$\Phi(\mathbf{p})=\int_{\mathbf{q}_{1} \cdots \mathbf{q}_{m}} h\left(\mathbf{p},-\mathbf{q}_{1} \cdots-\mathbf{q}_{m}\right) \phi\left(\mathbf{q}_{1}\right) \cdots \phi\left(\mathbf{q}_{m}\right)$,
$\Pi(\mathbf{p})=-m \int_{\mathbf{q}_{1} \cdots \mathbf{q}_{m}} h\left(-\mathbf{q}_{1}, \mathbf{p}, \cdots-\mathbf{q}_{m}\right) \pi\left(\mathbf{q}_{1}\right) \phi\left(\mathbf{q}_{2}\right) \cdots \phi\left(\mathbf{q}_{m}\right)$.

The canonical commutation relations (CCR) still hold under the unitary, albeit nonlinear, transformation of the fields (11), $[\tilde{\phi}(\mathbf{p}), \tilde{\pi}(\mathbf{q})]=i \bar{\delta}(\mathbf{p}+\mathbf{q})$. Noticing that the Gaussian cMERA is generated by the quadratic operator (2), it is clear that operators $\mathcal{B}$ which are linear or quadratic in $\pi$ 's and $\phi$ 's do not yield any improvement upon the Gaussian ansatz. Therefore, in going beyond, one must consider operators $\mathcal{B}$ that at least are cubic in the products of these fields.
In terms of wave functionals, the action of $U_{N G}$ on a functional $A[\phi]$ can be understood as a nonlinear shifting of the argument from $\phi$ to $\phi-s \phi^{m}$ and thus, for the Gaussian wave functional, $U_{N G} \Psi_{G}[\phi]=\Psi_{G}\left[\phi-s \phi^{m}\right][17,18]$.

Hence, our proposal to build nonperturbative cMERA states for interacting field theories is based on the idea of defining the set of scale-dependent nonlinear transformations

$$
\begin{aligned}
& \tilde{\phi}(\tilde{\mathbf{p}})=U_{N G}(u)^{\dagger} \phi(\mathbf{p}) U_{N G}(u), \\
& \tilde{\pi}(\tilde{\mathbf{p}})=U_{N G}(u)^{\dagger} \pi(\mathbf{p}) U_{N G}(u),
\end{aligned}
$$

where $U_{N G}(u) \equiv U_{N G} U_{S G}(u)$ and $U_{S G}(u) \equiv U_{S} U_{G}\left(u, u_{\mathrm{IR}}\right)$. As commented above, in going beyond the Gaussian approach, for $U_{N G}$ one must consider operators $\mathcal{B}$ that at least are cubic in the products of these fields. Here we will focus in the simplest one [29], i.e., the case $m=2$ which we denote by $\mathcal{B}=\pi \phi^{2}$ and explicitly reads

$\mathcal{B}=-s \int_{\mathbf{p q}_{1}, \mathbf{q}_{2}} g\left(p, q_{1}, q_{2}\right) \pi(\mathbf{p}) \phi\left(\mathbf{q}_{1}\right) \phi\left(\mathbf{q}_{2}\right) \bar{\delta}\left(\mathbf{p}+\mathbf{q}_{1}+\mathbf{q}_{2}\right)$,

where, from a cMERA point of view, $g\left(p, q_{1}, q_{2}\right)$ can be interpreted as a variational coupling-dependent momentum cut-off function [24]. With this choice for $\mathcal{B}$, the transformed fields result

$$
\begin{aligned}
& \tilde{\phi}(\tilde{\mathbf{p}})=\Sigma_{(-)}\left(\tilde{p} e^{u} ; u\right)\left(\phi(\tilde{\mathbf{p}})+s e^{\frac{d}{2} u} \Phi(\tilde{\mathbf{p}})\right), \\
& \tilde{\pi}(\tilde{\mathbf{p}})=\Sigma_{(+)}\left(\tilde{p} e^{u} ; u\right)\left(\pi(\tilde{\mathbf{p}})-2 s e^{\frac{d}{2} u} \Pi(\tilde{\mathbf{p}})\right),
\end{aligned}
$$

where we have made the change of variables in momenta $\mathbf{p} \equiv e^{u} \tilde{\mathbf{p}} . \quad$ In addition, we have defined $\Sigma_{( \pm)}(p ; u) \equiv$ $e^{ \pm f(p, u)} e^{-\frac{d}{2} u}$ and

$\Phi(\tilde{\mathbf{p}})=\int_{\tilde{\mathbf{q}}_{1} \tilde{\mathbf{q}}_{2}} \tilde{g}\left(\tilde{p}, \tilde{q}_{1}, \tilde{q}_{2}\right) \phi\left(\tilde{\mathbf{q}}_{1}\right) \phi\left(\tilde{\mathbf{q}}_{2}\right) \delta\left(\tilde{\mathbf{p}}-\tilde{\mathbf{q}}_{1}-\tilde{\mathbf{q}}_{2}\right)$,

$\Pi(\tilde{\mathbf{p}})=\int_{\tilde{\mathbf{q}}_{1} \tilde{\mathbf{q}}_{2}} \tilde{g}\left(\tilde{q}_{1}, \tilde{p}, \tilde{q}_{2}\right) \pi\left(\tilde{\mathbf{q}}_{1}\right) \phi\left(\tilde{\mathbf{q}}_{2}\right) \delta\left(\tilde{\mathbf{p}}-\tilde{\mathbf{q}}_{1}-\tilde{\mathbf{q}}_{2}\right)$,

where the scale-transformed non-Gaussian variational cutoff is given by

$\tilde{g}\left(\tilde{p}, \tilde{q}_{1}, \tilde{q}_{2}\right) \equiv e^{f\left(\tilde{p} e^{u}, u\right)-f\left(\tilde{q}_{1} e^{u}, u\right)-f\left(\tilde{q}_{2} e^{u}, u\right)} g\left(\tilde{p} e^{u}, \tilde{q}_{1} e^{u}, \tilde{q}_{2} e^{u}\right)$.

That is to say, as it occurs in the standard cMERA formulation, the variational parameters explicitly depend on the scale transformation. Hence, the cMERA scaledependent wave functional $\Psi_{N G}[\phi ; u]=U_{N G} \Psi_{G}[\phi ; u]$ is given by

$$
\Psi_{N G}[\phi ; u]=\Psi_{G}\left[\Sigma_{(-)}\left(\tilde{p} e^{u} ; u\right)\left(\phi(\tilde{\mathbf{p}})-s e^{\frac{d}{2} u} \Phi(\tilde{\mathbf{p}})\right)\right],
$$


where we have assumed, for simplicity, that $\chi_{0}=0$. Regarding the solution of the Gaussian variational parameter $f(p ; u)$ given in [2,3], it is straightforward to see that $\left.\Sigma_{( \pm)}\left(\tilde{p} e^{u} ; u\right)\right|_{u \rightarrow 0}=1$ and thus, (16) reduces to (11) and $\Psi_{N G}[\phi ; 0]=\Psi_{G}[\phi(\mathbf{p})-s \Phi(\mathbf{p})]$.

\section{NON-GAUSSIAN CORRELATION FUNCTIONS}

As in the Gaussian case, the non-Gaussian cMERA based on the $\pi \phi^{2}$ presented here is specially well suited to analyze correlation functions. These observables distinguish the ground states of interacting theories from those of noninteracting ones: i.e., while for Gaussian states the connected correlation functions of order higher than two vanish, those of interacting systems are generally nonzero. In addition, the multiscale approach provides a procedure to gain some understanding of the nonperturbative effects taking place at different scales.

From (16), we write the following structure of the $n$-point correlators at scale $u$ in real space

$$
\begin{aligned}
G^{(n)}\left(\mathbf{x}_{1}, \ldots, \mathbf{x}_{n}\right) \equiv & \left\langle\phi_{1} \cdots \phi_{n}\right\rangle_{N G} \\
= & \left\langle\phi_{1} \cdots \phi_{n}\right\rangle_{G} \\
& +s\left[\left\langle\Phi_{1} \phi_{2} \cdots \phi_{n}\right\rangle_{G}+\cdots+\left\langle\phi_{1} \cdots \phi_{n-1} \Phi_{n}\right\rangle_{G}\right] \\
& +s^{2}\left[\left\langle\Phi_{1} \Phi_{2} \phi_{3} \cdots \phi_{n}\right\rangle_{G}+\cdots+\left\langle\phi_{1} \cdots \Phi_{n-1} \Phi_{n}\right\rangle_{G}\right] \\
& \vdots \\
& +s^{n}\left\langle\Phi_{1} \cdots \Phi_{n}\right\rangle_{G}
\end{aligned}
$$

where $\phi_{i} \equiv \phi\left(\mathbf{x}_{i}\right)$ and $\Phi_{j} \equiv \Phi\left(\mathbf{x}_{j}\right)$. The correlation functions break up into interactionless disconnected functions and connected ones containing information about the interaction. The first four connected functions are

$$
\begin{aligned}
G_{c}^{(1)}\left(\mathbf{x}_{1}\right) & =s \tilde{\chi}_{1}, \\
G_{c}^{(2)}\left(\mathbf{x}_{1}, \mathbf{x}_{2}\right) & =\tilde{D}(12)+s^{2} \tilde{\chi}_{2}(12), \\
G_{c}^{(3)}\left(\mathbf{x}_{1}, \mathbf{x}_{2}, \mathbf{x}_{3}\right) & =s\left[\tilde{\chi}_{3}\right]_{(123)}+s^{3} \tilde{\chi}_{4}(12,23,31), \\
G_{c}^{(4)}\left(\mathbf{x}_{1}, \mathbf{x}_{2}, \mathbf{x}_{3}, \mathbf{x}_{4}\right) & =\frac{s^{2}}{2}\left[\tilde{\chi}_{5}\right]+s^{4}\left(\left[\tilde{\chi}_{2} \tilde{\chi}_{2}\right]+\left[\tilde{\chi}_{6}\right]\right),
\end{aligned}
$$

where we use the notation $a b \equiv \mathbf{x}_{a b} \equiv \mathbf{x}_{a}-\mathbf{x}_{b} . \tilde{D}(a b) \equiv$ $D(a b ; u)$ is the scale-dependent propagator

$$
\tilde{D}(a b)=\frac{1}{2} \int_{\mathbf{p}} e^{-2 f(p, u)} F\left(p e^{-u}\right) e^{i \mathbf{p} \cdot \mathbf{x}_{a b}} .
$$

The loop integrals $\tilde{\chi}_{i}(\mathbf{x} ; u), i=1, \cdots 6$ depend both on the positions and the scale $u$ and their explicit expressions and bracketed quantities involving them can be found in [24].

Connected functions show how the non-Gaussian cMERA procedure goes beyond the Gaussian approximation and captures scale-dependent nonperturbative contributions, which are arranged in powers of the variational parameter $s$. Focusing on quantities that usually measure the non-Gaussianity of a system, we notice that the skewness, related with the 3-point function, is given by $\gamma_{1}^{2}(s ; u) \equiv \frac{\left(G_{c}^{(3)}(123)\right)^{2}}{\left(G_{c}^{(2)}\right)^{3}} \underset{s \rightarrow 0}{\sim} \frac{\left.\left(\tilde{X}_{3}\right]_{123}\right)^{2}}{[\tilde{D} \tilde{D} \tilde{D}]} s^{2}+\mathcal{O}\left(s^{4}\right)$, where $\left(G_{c}^{(2)}\right)^{3} \equiv$ $G_{c}^{(2)}(12) G_{c}^{(2)}(13) G_{c}^{(2)}(23)$. In the limit of large $s(s \rightarrow \infty)$, the skewness achieves the limiting value $\gamma_{1, \infty}^{2} \sim$ $\tilde{\chi}_{4}^{2}(12,23,31) /\left[\tilde{\chi}_{2} \tilde{\chi}_{2} \tilde{\chi}_{2}\right]+\mathcal{O}\left(s^{-2}\right)$. In this sense, the quantities that usually can be measured in the experiments are the full and connected 2-point and 4-point correlation functions, as well as the point-dependent excess kurtosis over a Gaussian model [25]. For the latter, we obtain,

$$
\gamma_{2}(s ; u) \equiv \frac{G_{c}^{(4)}(1234)}{\left[G_{c}^{(2)} G_{c}^{(2)}\right]} \underset{s \rightarrow 0}{\sim} \frac{\left[\tilde{\chi}_{5}\right]}{2[\tilde{D} \tilde{D}]} s^{2}+O\left(s^{4}\right),
$$

where $\left[G_{c}^{(2)} G_{c}^{(2)}\right]=G_{c}^{(2)}(12) G_{c}^{(2)}(34)+G_{c}^{(2)}(13) G_{c}^{(2)}(24)+$ $G_{c}^{(2)}(14) G_{c}^{(2)}(23)$. In the limit of strong non-Gaussianity, $s \rightarrow \infty$, the excess kurtosis goes to a limiting value $\gamma_{2, \infty} \sim 1+\left[\tilde{\chi}_{6}\right] /\left[\tilde{\chi}_{2} \tilde{\chi}_{2}\right]+\mathcal{O}\left(s^{-2}\right)$.

\section{EQUATIONS FOR THE VARIATIONAL PARAMETERS}

We remark that to fully solve the non-Gaussian cMERA tensor network and evaluate the previous expressions for a theory with a Hamiltonian $\mathcal{H}$, we must obtain the optimal values for the variational parameters $F(p), g\left(p, q_{1}, q_{2}\right)$ and $s$. This is addressed by minimizing the expectation value of the energy density $\langle\mathcal{H}\rangle=\left\langle\Psi_{G}\left|U_{N G}^{\dagger} \mathcal{H} U_{N G}\right| \Psi_{G}\right\rangle$ at some length scale $u$, that in our case is the UV limit, (i.e., $u \rightarrow 0$ ). Here, we discuss two different theories. First we consider the $\lambda \phi^{4}$ scalar theory whose Hamiltonian density reads $\mathcal{H}_{\phi^{4}}=\mathcal{H}_{\text {kin }}+\frac{1}{2} m^{2} \phi(x)^{2}+\frac{\lambda}{4 !} \phi(x)^{4}$, where $\mathcal{H}_{\text {kin }}=$ $1 / 2\left(\pi(x)^{2}+[\nabla \phi(x)]^{2}\right)$ and $m$ and $\lambda$ are the bare mass and the bare coupling respectively. As any other polynomial 
interaction is a straightforward extension of this work, we also discuss a non-power-like potential, such as the sine-Gordon model whose Hamiltonian is given by $\mathcal{H}_{\mathrm{sG}}=\mathcal{H}_{\text {kin }}-\frac{\alpha}{\beta^{2}}[\cos \beta \phi(x)-1]$, in which $\beta$ is a dimensionless parameter, while $\alpha$ can be regarded as the square of the bare mass in the case of vanishing $\beta$. The energy expectation value of the $\lambda \phi^{4}$ theory is

$$
\begin{aligned}
\left\langle\mathcal{H}_{\phi^{4}}\right\rangle= & \left\langle\mathcal{H}_{\mathrm{kin}}\right\rangle+\frac{1}{2} m^{2}\left(s^{2} \chi_{2}+\phi_{c}^{2}\right) \\
& +\frac{\lambda}{4 !}\left[3 I^{2}+6 s^{2}\left(I \chi_{2}+\chi_{5}\right)+3 s^{4}\left(\chi_{2}^{2}+\chi_{6}\right)\right. \\
& \left.+4 \phi_{c}\left(3 s \chi_{3}+s^{3} \chi_{4}\right)+6 \phi_{c}^{2}\left(I+s^{2} \chi_{2}\right)+\phi_{c}^{4}\right],
\end{aligned}
$$

where $\phi_{c}=\chi_{0}+s \chi_{1},\left\langle\mathcal{H}_{\mathrm{kin}}\right\rangle=\frac{1}{4} \int_{\mathbf{p}}\left[F(p)^{-1}+p^{2} F(p)\right]+s^{2} \chi_{7}$ and $I=1 / 2 \int_{\mathbf{p}} F(p)$. The notation $\chi_{i}$ means that the loop integrals are evaluated at the same spatial point $\mathbf{x}$, i.e., $\chi_{i} \equiv \tilde{\chi}_{i}\left(\mathbf{x}_{a b}=0 ; u=0\right)$.

The equations for the optimal values of the variational parameters $s, F(p)$ and $g\left(p, q_{1}, q_{2}\right)$ are obtained, for a fixed $\phi_{c}$, by deriving $\left\langle\mathcal{H}_{\phi^{4}}\right\rangle$ with respect to them and then equating to zero [24]. This yields a set of nonlinear coupled equations that must be self-consistently and numerically solved. However, our aim here is to provide expressions that explicitly show the relation between the variational parameters and the coupling constants of the models under consideration. To proceed, we note that $\left\langle\mathcal{H}_{\phi^{4}}\right\rangle$ and their related optimization equations greatly simplify for $\phi_{c} \sim 0$ where the kernel $F(p)$ reduces to $F(p)=$ $1 / \sqrt{p^{2}+\mu^{2}}+\mathcal{O}\left(\phi_{c}^{2}\right)$, with $\mu$ a variational parameter $[21,24]$. In that case, $\mu^{2}=m^{2}+(\lambda / 2) I_{0}\left(\mu^{2}\right)$, where $I_{0}\left(\mu^{2}\right)=\frac{1}{2} \int_{\mathbf{p}}\left(p^{2}+\mu^{2}\right)^{-\frac{1}{2}}$. Further, we note that only the product $s g$ is meaningful and thus, fixing $s$ to be $s=-4 \lambda \phi_{c}$ is a way to conveniently normalize $g$ [30]. Finally, denoting $f(\mathbf{p}, \mathbf{q}) \equiv g(|\mathbf{p}+\mathbf{q}|, p, q)$, the optimal cutoff function is the solution of

$f(\mathbf{p}, \mathbf{q})=G(\mathbf{p}, \mathbf{q})\left(1-4 \lambda \int_{\mathbf{k}}[f(\mathbf{p}, \mathbf{k})+f(\mathbf{q}, \mathbf{k})] F(k)\right)$,

with $G(\mathbf{p}, \mathbf{q})$ a combination of kernels given in [24]. The term proportional to $s \chi_{3}$ in (22) is the major contribution to the improvement of the energy value compared to the Gaussian estimate [17,18]. Indeed, the optimal $\chi_{3}$ (given in terms of the solution of (23) is seen to contain an infinite series of diagrammatic contributions to the two-point function that are complementary to the "cactus"-diagrams resummation [20]. This highlights to what extent, the trial wave functionals of the non-Gaussian cMERA, may produce approximations that go far beyond the Gaussian approximation. Remarkably, the NLCT procedure in $d=1$ includes more physics but no further infinities than those posed by the cactus-diagrams. However, the renormalization of the non-Gaussian variational calculations in $d>1$ is shown to be much more involved and the contributions generated by the NLCT need infinite rescalings of the bare parameters [18].

Regarding the sine-Gordon model, when computing $\left\langle\mathcal{H}_{\mathrm{sG}}\right\rangle$, the term $\langle\cos \beta \phi\rangle$ poses a challenge to the NLCT method as this interaction term is nonpolynomial. In [24] it is shown that when the momentum support of the $p$-modes in $g\left(p, q_{1}, q_{2}\right)$ is sufficiently small in comparison with the support of the $q$-modes, one may write $\langle\cos \beta \phi\rangle=$ $\exp \left(-\beta^{2} / 2 I_{0}\left(\mu^{2}\right)\right) \cos \beta \varphi_{c}$ with $\varphi_{c}=s \chi_{1}$ and $\mu$ a variational mass parameter. In this limit, the optimization procedure can be applied to this model.

\section{DISCUSSION}

In this work, a general method for building non-Gaussian generalizations of the cMERA has been presented. The method uses a class of nonlinear canonical transformations which are then applied to a Gaussian wave functional. We have shown how to obtain nonperturbative effects on the correlation functions far beyond the Gaussian approximation in two scalar field theories. We expect this can be useful in addressing recent experimental data on higher order correlation functions in many body systems $[25,26]$. Furthermore, our method shows how the cMERA formalism could provide a systematic UV regularization scheme for generic interacting QFTs. In this sense, our approach can be generalized to fermionic and gauge field theories. In particular, we propose the following fermionic transformation acting on a spinor $\psi(\mathbf{k})$ :

$$
\begin{aligned}
\mathcal{B}= & \int_{\mathbf{p q}_{1} \cdots \mathbf{q}_{l}} g^{\alpha \beta_{1} \cdots \beta_{l}}\left(\mathbf{p}, \mathbf{q}_{1}, \ldots, \mathbf{q}_{l}\right) \pi_{\alpha}(\mathbf{p}) \\
& \times \psi_{\beta_{1}}\left(\mathbf{q}_{1}\right) \cdots \psi_{\beta_{l}}\left(\mathbf{q}_{l}\right) \delta\left(\mathbf{p}+\mathbf{q}_{1}+\cdots+\mathbf{q}_{l}\right),
\end{aligned}
$$

where Greek indices denote spinor components, $g$ is a variational (non-)Grassmannian function and $\pi_{\alpha}(\mathbf{p}) \equiv$ $\delta / \delta \psi_{\alpha}(-\mathbf{p})$ is the conjugate momentum. Despite this transformation also truncates, a model-dependent analysis, which is beyond the scope of this paper, would impose additional restrictions on the indices $\beta_{i}$. We expect this transformation to be useful in addressing relevant physical phenomena in strongly coupled theories including chiral field theories.

Regarding dynamical settings such as quantum quenches, the method promises to be useful as for the moment, all studies with the Gaussian cMERA, assume that the time-evolved state after the quench remains Gaussian along the evolution. Finally, it is worthwhile to explore what geometrical interpretation can be found for the non-Gaussian cMERA ansatz presented in this work. 


\section{ACKNOWLEDGMENTS}

J. J. F. M. acknowledges A. Bhattacharyya and T. Takayanagi for useful discussions and comments. J. M. V. thanks J. Cotler and M. Muller for many fruitful discussions. The work of J. J. F. M. is supported by Universidad de Murcia. J. M. V. is supported by Ministerio de Economia y
Competitividad FIS2015-69512-R and Programa de Excelencia de la Fundacion Seneca 19882/GERM/15. J. J.F. M. and E. T. L. acknowledge the financial support of Spanish Ministerio de Economia y Competitividad and CARM Fundación Seneca under Grants No. FIS201528521 and No. 21257/PI/19.
[1] G. Vidal, Phys. Rev. Lett. 99, 220405 (2007).

[2] J. Haegeman, T. J. Osborne, H. Verschelde, and F. Verstraete, Phys. Rev. Lett. 110, 100402 (2013).

[3] M. Nozaki, S. Ryu, and T. Takayanagi, J. High Energy Phys. 10 (2012) 193.

[4] A.Franco-Rubio and G.Vidal, J. High Energy Phys. 12 (2017) 129.

[5] M. Miyaji, T. Numasawa, N. Shiba, T. Takayanagi, and K. Watanabe, Phys. Rev. Lett. 115, 171602 (2015).

[6] M. Miyaji, T. Takayanagi, and K. Watanabe, Phys. Rev. D 95, 066004 (2017).

[7] A. Mollabashi, M. Nozaki, S. Ryu, and T. Takayanagi, J. High Energy Phys. 03 (2014) 98.

[8] P. Caputa, N. Kundu, M. Miyaji, T. Takayanagi, and K. Watanabe, Phys. Rev. Lett. 119, 071602 (2017).

[9] J. Molina-Vilaplana, J. High Energy Phys. 09 (2015) 2.

[10] J. Molina-Vilaplana, Phys. Lett. B 755, 421 (2016).

[11] J. S. Cotler, J. Molina-Vilaplana, and M. T. Mueller, arXiv: 1612.02427.

[12] J. Cotler, M. R. M. Mozaffar, A. Mollabashi, and A. Naseh, Phys. Rev. D 99, 085005 (2019).

[13] C. S. Hsue, H. Kümmel, and P. Ueberholz, Phys. Rev. D 32, 1435 (1985).

[14] U. Kaulfuss and M. Altenbokum, Phys. Rev. D 35, 609 (1987).

[15] M. Funke, U. Kaulfuss, and H. Kümmel, Phys. Rev. D 35, 621 (1987).

[16] D. Horn and M. Weinstein, Phys. Rev. D 30, 1256 (1984).
[17] L. Polley and U. Ritschel, Phys. Lett. B 221, 44 (1989).

[18] U. Ritschel, Z. Phys. C 47, 457 (1990).

[19] U. Ritschel, Z. Phys. C 51, 469 (1991).

[20] U. Ritschel, Z. Phys. C 63, 345 (1994).

[21] R. Ibañez-Meier, A. Mattingly, U. Ritschel, and P. M. Stevenson, Phys. Rev. D 45, 2893 (1992).

[22] P. M. Stevenson, Phys. Rev. D 32, 1389 (1985).

[23] J. Cotler, M. R. M. Mozaffar, A. Mollabashi, and A. Naseh, arXiv:1806.02831.

[24] See Supplemental Material at http://link.aps.org/ supplemental/10.1103/PhysRevD.100.065025 for further details.

[25] I. Kukuljan, S. Sotiriadis, and G. Takacs, Phys. Rev. Lett. 121, 110402 (2018).

[26] T. Schweigler, V. Kasper, S. Erne, I. Mazets, B. Rauer, F. Cataldini, T. Langen, T. Gasenzer, J. Berges, and J. Schmiedmayer, Nature (London) 545, 323 (2017).

[27] $\operatorname{Ad}_{\mathcal{B}}(A) \equiv \exp (\mathcal{B}) A \exp (-\mathcal{B}), \operatorname{ad}_{\mathcal{B}}(A) \equiv[\mathcal{B}, A]$

[28] In [23], an analysis of the Bender-Dunne basis has been done and some nonlinear transformations are proposed. Because nested commutators with $n \geq 2$ induce operators of powers higher than 4 , the algebra closes perturbatively. Hence, their construction is done up to $\mathcal{O}(\lambda)$, namely for weak coupling regimes.

[29] Namely, the NLCT method allows to build a set of nonGaussian cMERA tensor networks, each one based on different choices of $\mathcal{B}=\pi \phi^{m}$, with $m \in \mathbb{N}$.

[30] This relation does not impose any restriction on $\lambda$ which, in particular, can be finite. 